\title{
Multimodality Monitoring: Toward Improved Outcomes
}

\author{
Richard Gandee, DO ${ }^{1}$ Chad Miller, MD, FNCS ${ }^{2}$ \\ ${ }^{1}$ Internal Medicine, Doctors Hospital, OhioHealth, Columbus, Ohio \\ ${ }^{2}$ Riverside Methodist Hospital, OhioHealth, Columbus, Ohio \\ Address for correspondence Chad Miller, MD, FNCS, Riverside \\ Methodist Hospital, OhioHealth, 3535 Olentangy River Road, \\ Columbus, OH 43214 (e-mail: chad.miller2@ohiohealth.com).
}

Semin Respir Crit Care Med 2017;38:785-792.

\begin{abstract}
Keywords

- multimodality monitoring

- neurocritical care

- brain injury

- extraventricular drain

- transcranial Doppler

- Licox

- CEEG

Multimodality monitoring provides insights into the critically ill brain-injured patient through the assessment of biochemical, physiological, and electrical data that provides insight into a patient's condition and what strategies may be available to limit further damage and improve the odds for recovery. Modalities utilized include evaluation of intracranial pressure along with cerebral perfusion pressure to determine adequate blood flow; continuous electroencephalography to protect the patient from seizures and to identify early functional manifestations of ischemia and toxicity; transcranial Doppler evaluation for bedside review of circulatory adequacy; tissue oxygen monitoring to establish that brain tissue is receiving adequate oxygen from blood flow; and microdialysis to evaluate the metabolic function of the tissue in areas of concern. These monitors provide insights regarding specific aspects of brain tissue and overall brain function in the critically ill patient. Although recommendations continue to evolve for therapeutic targets for each of these modalities, an effective clinician may use each of these modalities to evaluate patients on an individual basis to improve the outcome of each patient, tailoring management to provide the care needed for any unique clinical presentation.
\end{abstract}

Today's critical care clinician has a plethora of options for monitoring the critically ill patient, specifically those patients with brain injuries at risk for further deterioration related to impaired blood flow, metabolism, or oxygenation. Monitoring for the evolution of the primary injury and the development of secondary injuries to the brain is paramount in the evaluation, treatment, and prognosis of any neurocritical care patient. The evidence of such injury may require specific monitoring with one of several techniques, and it is therefore essential to be familiar with the basics of the various types of monitoring available for the brain-injured patient.

\section{Intracranial Pressure}

Intracranial pressure (ICP) results from the volumes of three intracranial components: brain parenchyma, cerebral blood volume, and the volume of cerebral spinal fluid. The combination of these three components creates a near constant sum that is subject to an autoregulatory system that can maintain adequate cerebral blood flow despite variations in pressure resulting from these three components. ${ }^{1}$ In the critically ill patient, brain trauma or cerebral vascular injury can result in swelling that increases the ICP to a point that cerebral blood flow is compromised, resulting in the possibility of ischemia. The normal ICP range in healthy adults is typically 7 to $15 \mathrm{~mm} \mathrm{Hg}$. As ICP approaches 20 to $25 \mathrm{~mm} \mathrm{Hg}$, the brain's autoregulatory mechanisms may be overcome or impaired. Consistent values above $20 \mathrm{~mm} \mathrm{Hg}$ are associated with worse outcomes. ${ }^{2}$ In addition to the values obtained from ICP pressure monitors, the ICP waveform can be evaluated for changes. ${ }^{3}$ There are peaks along the ICP waveform: $\mathrm{P} 1, \mathrm{P} 2$, and P3. P1, the first peak is indicative of arterial
Issue Theme Advancements in Neurocritical Care and Emergency Neurology; Guest Editors: David Y. Hwang, MD, FNCS, and David M. Greer, MD, MA, FCCM, FAHA, FNCS, FAAN, FANA
Copyright $\odot 2017$ by Thieme Medical Publishers, Inc., 333 Seventh Avenue, New York, NY 10001, USA.

Tel: +1(212) 584-4662.
DOI https://doi.org/ 10.1055/s-0037-1608774. ISSN 1069-3424. 
pulsation; P2 (tidal wave), which follows P1, results from reflection of the arterial pulse and may be indicative of brain compliance; P3, the final wave is the result of aortic valve closure. In nonpathological waveforms, P1 is higher than P2. However, if brain parenchyma becomes less compliant, potentially due to swelling, the P2 wave will appear higher than P1, or may join P1 as one combined and broader peak. Recognition of the waveform morphology is complimentary to the analysis of absolute ICP elevation. ${ }^{4}$

ICP can be evaluated noninvasively utilizing transcranial Doppler (TCD) ultrasonography, pupillometry, and optic nerve sheath diameter measurement with ultrasound. Invasive methods include the use of external ventricular drains (EVDs) or intraparenchymal monitoring systems. There is debate regarding the accuracy of various methods of noninvasive ICP monitors. ${ }^{5}$ EVD allows for not only monitoring but also allows for therapeutic draining of cerebrospinal fluid (CSF). In addition, an EVD is the most accurate assessment of global ICP, and it has the advantage of limited measurement drift. ${ }^{6}$ However, ICP cannot be measured, while the EVD is set to drain. Furthermore, insertion and proper placement of an EVD in a patient who has compressed or altered ventricular anatomy may be challenging. Intraparenchymal pressure sensors can be inserted through a burr hole to a depth of 1.5 to $2.0 \mathrm{~cm}$ into brain parenchyma. ${ }^{7}$ While two particular sensors, the Codman and Neurovent, are compatible with magnetic resonance imaging, intraparenchymal sensors do not have the advantage of recalibration, cannot drain CSF, and are more expensive. ${ }^{3}$

Measurement of cerebral perfusion pressure (CPP) is valuable in the management of critically ill patients, and reflects the perfusing pressure of the brain with respect to $\mathrm{ICP}^{8} \mathrm{CPP}$ is the difference between the mean arterial pressure (MAP) and the ICP. The typical CPP in an adult is more than $50 \mathrm{~mm} \mathrm{Hg}$, but in the critically ill patient, current guidelines recommend maintaining a CPP between 60 and $70 \mathrm{~mm} \mathrm{Hg} .{ }^{9} \mathrm{CPP}$ less than $60 \mathrm{~mm} \mathrm{Hg}$ is associated with poor outcomes. ${ }^{10}$ Utilization of CPP as a therapeutic target requires awareness of the patient's autoregulatory state. Impaired cerebral autoregulation may last up to 2 weeks following severe brain injury. ${ }^{11}$ The loss of the autoregulatory mechanisms may result in the need for increased MAPs to maintain a desired level of CPP, which may in turn, result in systemic complications, such as acute respiratory distress syndrome. $^{12}$

Both ICP and CPP are commonly utilized as management targets following brain injury. 2,6,9 Management dictated by a single value has been shown to be less efficacious than management utilizing both values. ${ }^{13}$ Despite the reliance on ICP and CPP for monitoring, the 2012 BEST TRIP trial indicated that ICP targeted management aimed at maintaining the ICP at $20 \mathrm{~mm} \mathrm{Hg}$ or less was not superior to imaging and clinical examination in treating patients with traumatic brain injury (TBI). ${ }^{14,15}$ Despite the lack of ICP monitoring, it is noteworthy that the control group received particularly aggressive ICP lowering therapies, and that surgical and critical care management deviated from traditional methods. Nonetheless, the utility of ICP monitoring as a sole therapeutic target remains in question. ${ }^{16}$ ICP elevations may result from a variety of anatomic and pathophysiologic causes. Therefore, the use of additional monitoring modalities may provide insight as to the cause of intracranial hypertension.

\section{Electroencephalogram}

In critically ill patients, particularly those in a comatose state, continuous electroencephalogram EEG (cEEG) monitoring is a useful tool for detecting electrophysiologic changes. In the critical care setting, the physical examination or evidence of neurologic deterioration may be limited, and cEEG can provide insights into a patient's neurologic status that would otherwise be unavailable to the clinician. ${ }^{17}$ EEG can be used for detection of nonconvulsive seizures or ischemia, and may provide useful information regarding prognosis. Though the information available via cEEG is substantial, there are also several problems associated with the technology, including cost and the need to consistently check leads and monitor for possible interference in the critical care setting. Given the vast quantity of data, the cEEG may be more vulnerable to misinterpretation of the results. ${ }^{18}$

EEG is able to dynamically evaluate brain function by monitoring the electrical activity of the cortex using a noninvasive approach with placement of electrodes on the patient's scalp. The number of electrodes utilized has varying consequences. While a smaller number of electrodes provides flexibility to work around various other monitoring devices and a potential for increased efficiency in regard to application and maintenance, a larger, "full electrode" setup involving 16 or more electrodes helps refine the signal, thus reducing artifact, which may be a problem in the critical care setting. ${ }^{19}$ In addition, a full set of electrodes provides the possibility of a more refined localization of pathology, and a higher number of electrodes establishes a system in which interpretation may continue despite the loss of a single electrode that may occur when moving a patient or due to poor connection. ${ }^{20}$

Interpretation of EEG in the critically ill patient is very different from the ambulatory patient. As such, it may be difficult to identify patterns associated with potential longterm neurologic injury as opposed to those associated with transient metabolic and pharmacologic causes. For this reason, continuous EEG in the critical care setting is typically augmented with video monitoring, which may aid the clinician in determining if specific changes on an EEG are associated with seizure, or if they are simply artifact secondary to movement. ${ }^{20}$

While neuroimaging is able to display damage to brain parenchyma after an event has taken place, EEG may provide greater insights into the functional status of the brain. Advantages to EEG include its safety and ease of placement. There are limitations to EEG monitoring, typically due to interference, poor resolution, incomplete contact between the patient's scalp and the electrode, as well as interference from other electrical devices (though this can potentially be ameliorated with the placement of additional leads for refinement of the data). ${ }^{19}$ However, placement of intracortical electrodes 
(placed with an EVD or through a burr hole) has been trialed at a few centers in an effort to improve resolution and diagnostic sensitivity. ${ }^{21-24}$ While this is associated with increased risk of placement, small studies have revealed that there is greater rate of seizure detection and decreased interference. Further studies have revealed that placement of intracortical electrodes is associated with similar safety results as other invasive modalities of monitoring. ${ }^{25}$

Multimodality monitoring guidelines recommend EEG in patients with altered consciousness of unknown cause, acute brain injury, convulsive status epilepticus who have not returned to baseline at 60 minutes from administration of medication, and during both therapeutic hypothermia as well as 24 hours after rewarming. ${ }^{2}$ cEEG helps guide treatment for patients placed in a pharmacologic coma in an effort to treat increased ICP (- Fig. 1). ${ }^{26}$ cEEG monitoring in comatose patients should take place for at least 24 to 48 hours, as this duration of monitoring captures the majority of events. ${ }^{27,28}$ EEG patterns, such as total power and $\alpha$-delta ratios, have been shown to correlate with vasospasm in subarachnoid hemorrhage (SAH). Quantitative EEG compresses hours of EEG data into a spectral array that occupies a single screen. This allows for quick recognition of pattern changes.

While convulsive seizures are the classic subject of EEG studies, nonconvulsive seizures are more common in critical illness and are one impetus for cEEG monitoring. Altered mental status, especially in the critical care setting, should be evaluated with EEG in an effort to rule out nonconvulsive events. Typical causes include infections, toxins, metabolic abnormalities, withdrawal, and structural lesions. Undetected seizures are a source of secondary brain injury and have been associated with increased brain edema and mortality. While it is unclear if prognosis and recovery can be improved by treatment of subclinical seizures, their presence and persistence are closely tied to adverse outcomes.

Detection of cerebral ischemia is a lesser utilized application of cEEG. With reduction of cerebral blood flow, changes in EEG tracings typically occur within seconds. ${ }^{29}$ Loss of high frequencies and prominent slowing of the background EEG rhythm may occur when $\mathrm{CBF}$ decreases below 25 to 30 $\mathrm{mL} \cdot 100 \mathrm{~g}^{-1} \cdot \mathrm{min}^{-1}$. When CBF drops below 8 to $10 \mathrm{~mL} \cdot 100$ $\mathrm{g}^{-1} \cdot \mathrm{min}^{-1}$, which is low enough to cause irreversible cell death, all EEG frequencies are suppressed. ${ }^{30}$ Early ischemic detection with EEG analysis allows for earlier intervention.

Finally, EEG is an essential tool in titration of sedation therapy. In refractory status epilepticus and intracranial hypertension, EEG provides guidance to the proper dosing of medications to achieve burst suppression without concern for excessive sedation. ${ }^{31-33}$ Avoidance of excessive sedation results in a reduced risk of side effects and possible complications, such as ileus, hypotension, and immunosuppression. Studies also indicate that the use of continuous EEG in patients who have undergone therapeutic hypothermia may provide insight into a patient's prognosis earlier than the standard 72-hour time frame postwarming. ${ }^{34}$

\section{Transcranial Doppler}

TCD monitoring has expanded from a method for detecting vasospasm in patients with SAH to an effective and affordable method for evaluating cerebral blood flow, detecting

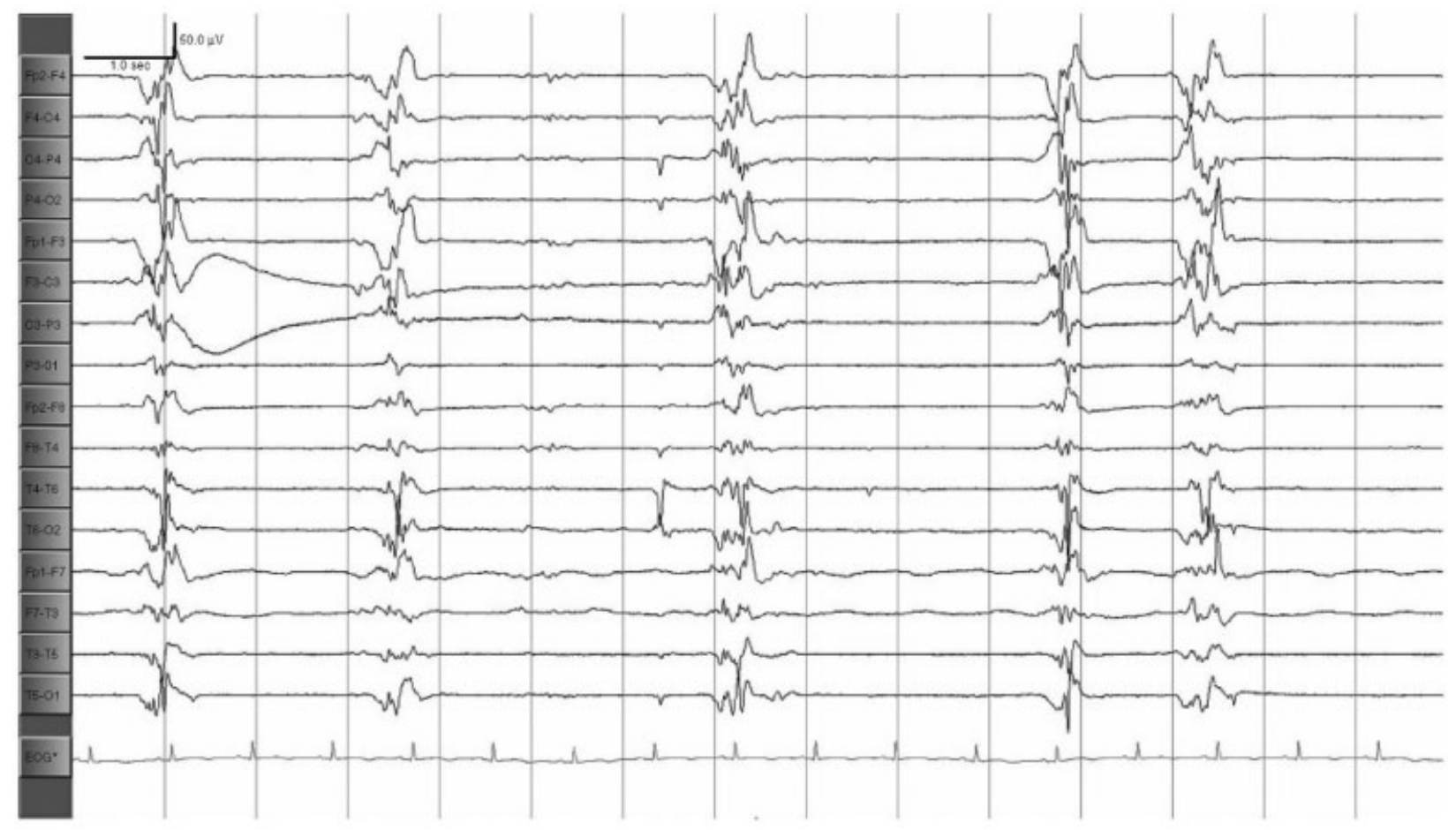

Fig. 1 Continuous electroencephalogram of patient receiving sedation for control of intracranial hypertension. A burst suppression pattern is shown resulting from therapeutic metabolic suppression. 
vascular stenosis, evaluating collateral flow, detecting embolization, and evaluating cerebral arterial patency. ${ }^{35,36}$ The advantages of TCD are that it can be performed at the bedside, is generally less expensive than other imaging modalities, and can be used for evaluation of both cerebral hemodynamics and monitoring for embolization of cerebral vessels. TCD can be used either intermittently or continuously and does not require intravascular contrast. The evaluation is performed using a $2-\mathrm{MHz}$ ultrasound wave produced by piezoelectric crystals that are stimulated electrically. ${ }^{35}$ The vessels are not directly imaged, but are visualized using the reflection of the ultrasound wave off of erythrocytes within the vessel imaged, which is then converted into an electric signal (-Fig. 2).

Training is required for use of TCD, and it is an operatordependent evaluation, unlike several other monitoring techniques. TCD transduction is limited by the skull, and cannot be acquired in a portion of patients due to skull thickness. ${ }^{35}$ There are four standard areas of the skull that provide acoustic windows into the brain. These areas are the temporal, orbital, suboccipital, and submandibular windows. Each window allows for the evaluation of different vessels. The middle cerebral (MCA), anterior cerebral (ACA), posterior cerebral, and posterior communicating arteries are visible through the transtemporal window. ${ }^{37}$ The transorbital window allows for visualization of the internal carotid artery (ICA) and occipital artery siphons. The space between the occipital bone and the atlas vertebrae allows for evaluation of the vertebral and basilar arteries. The cervical ICA can be evaluated through the submandibular window. The ICA and ACA may be difficult to evaluate with TCD alone, though this may be overcome with the addition of transcranial colorcoded duplex sonography. ${ }^{37}$

The two values that can be ascertained directly from sonography are peak systolic velocity (PSV; $\mathrm{cm} / \mathrm{s}$ ) and enddiastolic velocity (EDV; $\mathrm{cm} / \mathrm{s}$ ). PSV is the initial peak of the waveform of each cardiac cycle. Rapid upstroke is indicative

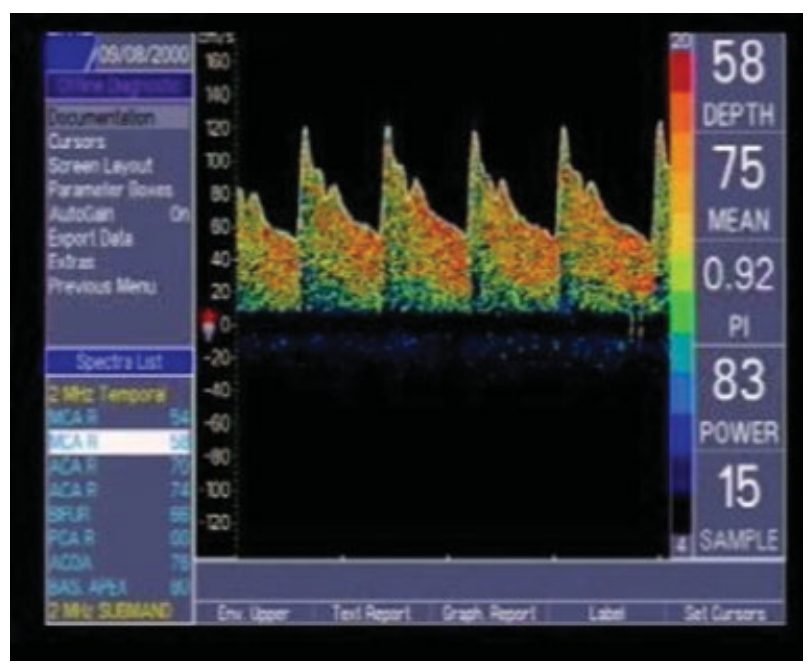

Fig. 2 Transcranial Doppler acquisition of a right middle cerebral artery waveform. Mean velocities and pulsatility indices are within normal limits for this study. of no severe stenotic lesion between the examined intracranial artery and the heart. EDV is typically 20 to $50 \%$ of the PSV, which indicates a low resistance intracranial arterial pattern of flow. ${ }^{37}$

Mean flow velocity (MFV; $\mathrm{cm} / \mathrm{s}$ ) is derived from the following: MFV $=\mathrm{EDV}+1 / 3$ (PSV - EDV). The MCA typically has the highest MFV of all major intracranial arteries. ${ }^{36}$ Flow resistance is evaluated using pulsatility index (PI; no units), calculated as follows: $\mathrm{PI}=(\mathrm{PSV}-\mathrm{EDV}) / \mathrm{MFV}$. A value more than 1.2 represents high resistance to blood flow, and more than 1.6 has been correlated with higher ICP values and worse outcomes. ${ }^{38}$ Finally, resistance index (RI) is another value for monitoring resistance but represents flow distal to the evaluated site. RI $=($ PSV - EDV $) / P S V$. Normal values are less than 0.75 .

In TCD, evaluation of the flow velocity is essential. Increases and decreases in flow velocity may represent narrowing of a vessel. As evaluation is limited to a specific vascular area, flow velocity changes at the site of evaluation may represent an occlusion proximal or distal to the site of evaluation. Likewise, a decrease in flow velocity may indicate vascular narrowing proximal to the site of evaluation but, if associated with an increased PI, may represent distal resistance to blood flow. ${ }^{35}$

In conjunction with other approaches to monitoring, such as cerebral tissue oxygenation, TCD may be able to help elucidate a vascular narrowing of impairment of flow that could inhibit the oxygenation of the brain. The advantage of TCD is that its ability to be performed at the bedside, which may allow for earlier identification of ischemia that might benefit from intervention.

In addition to dynamic evaluation of the cerebral vasculature of a patient at the bedside, TCD is a supplementary test that can be used in evaluating patients with sickle cell disease, acute thrombotic cerebrovascular occlusion, or perioperative monitoring in patients at high risk for embolization and infarction. ${ }^{35}$ TCD may also service as an ancillary test for brain death determination. Findings exist on a spectrum, from absence of flow to high resistance to diastolic flow reversal. Diastolic flow of zero (or negative) is not compatible with life, and is consistent with cerebral circulatory arrest; however, absence of signal could be secondary to operator/patient factors and is not acceptable for determination of brain death. When a complete neurologic assessment of brain death is not possible, TCD may provide data supportive of the clinical determination of brain death. ${ }^{39}$

\section{Brain Tissue Oxygen Monitoring}

Impairment of oxygen delivery to the brain is a common source of secondary brain injury. While other monitoring modalities are able to evaluate tissue oxygenation indirectly as a result of blood flow or metabolic changes that result from decreased oxygenation, novel techniques for monitoring cerebral tissue oxygenation include jugular venous saturation monitoring $\left(\mathrm{SjvO}_{2}\right)$, brain tissue oxygen tension, and near-infrared spectroscopy (NIRS). ${ }^{7}$ The advantage of these approaches is that oxygenation is directly measured, though each monitor varies 
in its technique and established normative values. Direct monitoring of brain oxygenation is recommended in patients undergoing therapeutic hyperventilation and any patient at risk for ischemia. ${ }^{2,6}$

$\mathrm{SjvO}_{2}$ may be used to monitor patients undergoing evaluation following TBI or SAH. A jugular venous catheter is placed above the level of $\mathrm{C} 1 / \mathrm{C} 2$ to diminish the potential of interference by blood return through the facial vein. ${ }^{40} \mathrm{SjvO}_{2}$ is a global assessment of brain oxygenation and may fail to identify focal regions of ischemia. Low jugular venous saturations may indicate decreased oxygen supply to the brain secondary to hypoperfusion or increased metabolic function requiring increased oxygen supply, resulting in increased uptake of oxygen. ${ }^{41}$ Increased oxygen levels may suggest excessive cerebral blood flow or decreased metabolic demand. Typical levels range from 60 to 75\%. Levels below $55 \%$ saturation are indicative of likely ischemia. ${ }^{40,42}$ Values more than $75 \%$ may indicate hyperemia, catheter misplacement, or decreased metabolic demand. ${ }^{43}$

NIRS is a noninvasive technique, capable of continuously monitoring cerebral oxygen saturation. This technique is limited to the cerebral cortex. NIRS is complicated by a wide variation in baseline ranges of regional oxygen saturation, as well as possible interference from the oxygen content of the skin, bones, and meninges, obscuring the accuracy of the results. ${ }^{7,44}$ In addition, pathological tissue changes may distort the results obtained using NIRS. Patients with TBI may not accurately reflect true tissue oxygenation due to the swelling associated with the trauma. Furthermore, extravascular blood, subdural air following neurosurgical intervention, and water between the skin and sensor may also impede the accuracy of tissue assessment.

$\mathrm{PbtO}_{2}$ monitoring attempts to measure oxygenation with a more regionally focused approach. $\mathrm{PbtO}_{2}$ utilizes the diffusion of oxygen through a membrane that is reduced at an electrode. A reference electrode is utilized for comparison, and the amount of oxygen is calculated as proportional to the difference in voltage between the reference and measurement electrodes. A temperature probe is included, as this process is temperature dependent. The temperature probe allows for correction of the changes in voltage based on changes in temperature. ${ }^{7}$ As the level of oxygen increases in the region, the rate of diffusion onto and reduction by the cathode will increase. ${ }^{3}$ Unfortunately, despite the ability to monitor local oxygenation, the sufficiency for cellular metabolism may remain unclear, as increased oxygen levels may simply reflect the inability of cells to appropriately utilize available oxygen. Values may be altered by levels of $\mathrm{CO}_{2}$ and $\mathrm{O}_{2}$ and by the presence of fevers, seizures, or shivering. ${ }^{45}$ Furthermore, as $\mathrm{PbtO}_{2}$ is a regional monitoring system, it requires appropriate and accurate placement of a catheter to evaluate the tissue in question (-Fig. 3). ${ }^{46}$ There are currently two systems available for monitoring of $\mathrm{PbtO}_{2}$, Licox, and Neurovent-PTO. While both are safe and effective, the systems provide assessment of cerebral oxygenation using different data output units. ${ }^{3}$ Current guidelines recommend intervention at a $\mathrm{PBtO}_{2}$ of less than $20 \mathrm{~mm} \mathrm{Hg}$.,

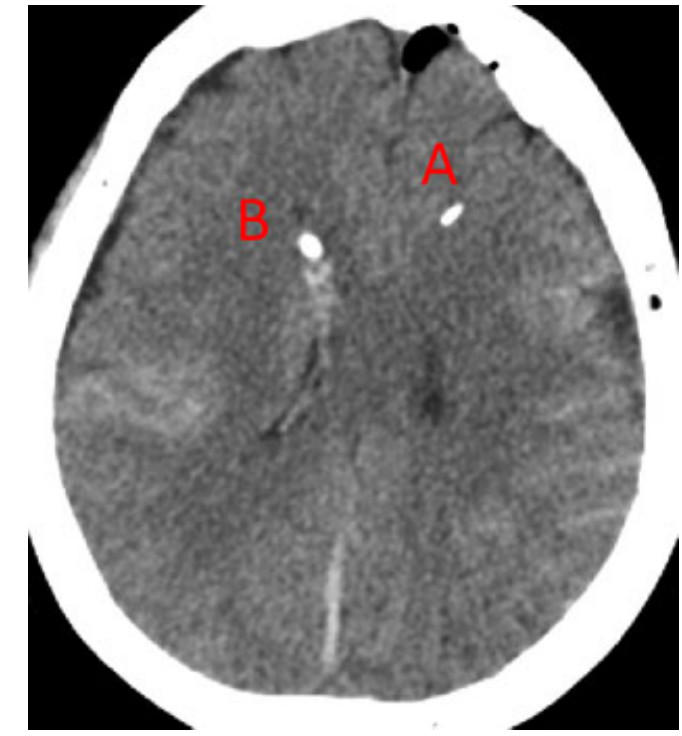

Fig. 3 Noncontrast head computed tomography of patient suffering aneurysmal subarachnoid hemorrhage. (A) The radiopaque tip of a brain tissue oxygen catheter as well as (B) a contralaterally placed extraventricular drain can be seen.

Among the three brain oxygenation monitors described, $\mathrm{PbtO}_{2}$ monitoring is the most frequently utilized due to its ability to monitor regions of interest. However, given the fact that even regional monitoring may not provide complete results, further evaluation of the metabolic substrates in a region may provide further insight into the status of a critically ill patient.

\section{Microdialysis}

Systemic markers of brain injury often poorly correlate with the health of brain metabolism. ${ }^{47-49}$ For this reason, the direct evaluation of interstitial brain metabolites may provide greater insight into the needs of the injured brain and risks for deterioration.

Cerebral microdialysis is an assessment tool that may be utilized to evaluate the metabolic substrates present in local tissue in real time. This evaluation may help in the early identification and potential prevention of secondary ischemic injury. In the critically ill patient, metabolic changes may signify greater tissue destruction and increased morbidity and mortality. The use of microdialysis in cerebral tissue utilizes the placement of a microdialysis catheter into the cerebral tissue in an area of concern (e.g., the ischemic penumbra following an ischemic event, a region identified as potentially ischemic or any region affected by trauma). The catheter is composed of a semipermeable membrane that allows diffusion of metabolic substrates from the interstitial fluid down a gradient into the catheter, which is initially filled only with an isotonic solution that allows for diffusion of substrates within the area of interest of quantitative analysis. ${ }^{3}$ The catheter is connected to a collection vial that may be removed so that the fluid can be analyzed. Ideally, samples can be taken at equal intervals and the fluid 
analyzed for concentrations. Substrates of particular interest include lactate, pyruvate, glucose, glycerol, and glutamate. Accurate assessment of the concentrations of these molecules is predicated on the flow rate through the catheter membrane and the size of the membrane, with slower flow rates and large catheters resulting in an assay of higher concentrations of solute..$^{50}$

As the major metabolic component of cellular energy, glucose regulation is keyed. Microdialysis monitoring may allow for evaluation of cerebral glucose levels, which can be helpful in evaluating the potential for recovery in a critically ill patient. Strict glycemic control in a critically ill patient may result in poor delivery of glucose to the brain and tissues in need of glucose for cellular repair, as increased metabolic substrates and products of metabolism without glucose can be cytotoxic and exacerbate primary damage suffered with an initial insult. ${ }^{50}$

Analysis of lactate and pyruvate concentrations may reveal risk for neuronal injury, but caution is necessary to monitor the ratio of lactate:pyruvate ratio, as lactate can be increased as a component of both anaerobic metabolism as well as normal cellular processes. As the availability of oxygen decreases and the cellular metabolism shifts to an anaerobic state, the level of lactate will increase as pyruvate is utilized as a cofactor in the anaerobic portion of glycolysis. In addition, a decrease in glucose delivery secondary to decreased blood flow will decrease the level of available pyruvate, and may alter the lactate:pyruvate ratio, highlighting the need to monitor more than just one chemical ratio. An increased ratio of lactate:pyruvate accompanied by a decrease in glucose may indicate the need for augmentation of cerebral perfusion or relaxation in the intensity of systemic glycemic control. If an increase in lactate is secondary to an increase in cellular metabolism, the ratio of lactate to pyruvate will likely remain stable. Ideally, the ratio of lactate:pyruvate is $\sim 20$; levels above 25 are indicative of impending metabolic damage. ${ }^{50}$

Glutamate concentrations are likewise difficult to interpret directly because glutamate is present in the interstitial fluid. Glutamate is a substrate of interest in patients who have experienced $\mathrm{SAH}$, ischemia, and cardiac arrest, since a rise in glutamate levels may be indicative of an inability of cells to take up interstitial glutamate due to cell death or poor energy supply. ${ }^{50-53}$ Typically, glutamate is taken up by cells in an energy-dependent manner that requires the metabolism of glucose. As glutamate levels increase, neuronal calcium channels have the potential to be opened as a direct result of increased glutamate concentrations. Opening of these calcium channels allows for the influx of calcium, leading to possible damage to the cellular membrane of the various cells of the brain. If levels of glutamate in the dialysate are increased, this may be an early indicator that cells are damaged and unable to properly function, or are not receiving adequate glucose to take up interstitial glutamate. Furthermore, glutamate is recognized as an earlier indicator of vasospasm when compared with other substrates, and an elevation may indicate the need to evaluate a patient for additional signs of vasospasm to prevent further damage. ${ }^{54}$
Glycerol is another substrate of particular interest, as it is a component of the fatty acids that make up cell membranes that may be disrupted in a patient with neurologic damage. Glucose typically provides sufficient energy to actively transport calcium out of cells. If glucose levels decrease, calcium may enter cells, which activates phospholipases to break down fatty acids. ${ }^{50}$ An increase in glycerol concentration is therefore an indication of potential breakdown of the cell membranes and an indicator of increasing cell damage. $^{21}$

Microdialysis is of particular interest in both SAH and TBI. In both clinical scenarios, the placement of the catheter is keyed to the evaluation of the dialysate. The catheter may be placed during surgery, transcutaneously, or through a cranial bolt. ${ }^{50}$ In SAH patients, the catheter should be placed in the tissue with the greatest potential risk for secondary ischemic injury (typically the region of the parent vessel). In TBI, catheters should not be placed in tissue that lacks viability, as analysis will not be reflective of therapeutic need or interventions. Instead, in a focal injury, one catheter may be placed in a region surrounding the tissue of concern, while another may be placed in normal tissue for comparison. If a patient has suffered diffuse TBI, the catheter should be placed in the right frontal region. ${ }^{50}$

Additional markers that may be directly evaluated through the use of microdialysis include reactive oxygen species, extracellular potassium concentration (which does not typically correlate with systemic levels of potassium), matrix metalloproteinase-9 levels, and interleukin-6 levels, which may function as further indicators of cellular injury, metabolic distress, poor prognosis, and delayed ischemia, respectively. ${ }^{55-57}$

\section{Conclusion}

The monitoring of any critically ill patient requires nearconstant evaluation and re-evaluation of myriad factors in an effort to improve the patient's prognosis. No single value or bit of data on its own can accurately portray or predict the condition of the critically ill patient. Multimodality monitoring provides the breadth of information that best illustrates the current state of a patient. While one test may provide a single measure, often more questions arise from the changing state of a patient's results. For this reason, the combination of these monitoring techniques provides a clearer elucidation of not only the objective changes in a patient but can provide the means to further investigate why those changes are taking place. Used in conjunction, these methods of evaluation may provide clinicians with the insight needed to properly address the specific needs of each patient and potentially improve outcomes.

\section{References}

1 Mokri B. The Monro-Kellie hypothesis: applications in CSF volume depletion. Neurology 2001;56(12):1746-1748

2 Le Roux P, Menon DK, Citerio G, et al. Consensus summary statement of the international multidisciplinary consensus conference on multimodality monitoring in neurocritical care: a 
statement for healthcare professionals from the Neurocritical Care Society and the European Society of Intensive Care Medicine. Neurocrit Care 2014;21(Suppl 2):S1-S26

3 Tasneem N, Samaniego EA, et al. Brain multimodality monitoring: a new tool in neurocritical care of comatose patient. Crit Care Res Pract Vol 2017;2017:6097265

4 Roh D, Park S. Brain multimodality monitoring: updated perspectives. Curr Neurol Neurosci Rep 2016;16(06):56

5 Kristiansson H, Nissborg E, Bartek J Jr, Andresen M, Reinstrup P, Romner B. Measuring elevated intracranial pressure through noninvasive methods: a review of the literature. J Neurosurg Anesthesiol 2013;25(04):372-385

6 Brain Trauma Foundation; American Association of Neurological Surgeons; Congress of Neurological Surgeons; Joint Section on Neurotrauma and Critical Care, AANS/CNS. Guidelines for the management of severe traumatic brain injury. VII. Intracranial pressure monitoring technology. J Neurotrauma 2007;24(1, suppl 1): S45-S54

7 Nordström C-H, Koskinen L-O, Olivecrona M. Aspects on the physiological and biochemical foundations of neurocritical care. Front Neurol 2017;8:274

8 Rosner MJ, Rosner SD, Johnson AH. Cerebral perfusion pressure: management protocol and clinical results. J Neurosurg 1995; 83(06):949-962

9 Brain Trauma Foundation. "Guidelines for Management of Severe Traumatic Brain Injury" 4th edition, 2016. Available at: http:// braintrauma.org/ coma/guidelines. Accessed on March 8, 2017

10 Andrews PJ, Sleeman DH, Statham PF, et al. Predicting recovery in patients suffering from traumatic brain injury by using admission variables and physiological data: a comparison between decision tree analysis and logistic regression. J Neurosurg 2002;97(02):326-336

11 Sviri GE, Aaslid R, Douville CM, Moore A, Newell DW. Time course for autoregulation recovery following severe traumatic brain injury. J Neurosurg 2009;111(04):695-700

12 Contant CF, Valadka AB, Gopinath SP, Hannay HJ, Robertson CS. Adult respiratory distress syndrome: a complication of induced hypertension after severe head injury. J Neurosurg 2001;95(04): 560-568

13 Gerber LM, Chiu YL, Carney N, Härtl R, Ghajar J. Marked reduction in mortality in patients with severe traumatic brain injury. J Neurosurg 2013;119(06):1583-1590

14 Jones S, Schwartzbauer G, Jia X. Brain monitoring in critically neurologically impaired patients. Int J Mol Sci 2016;18(01):43

15 Chesnut RM, Temkin N, Carney N, et al; Global Neurotrauma Research Group. A trial of intracranial-pressure monitoring in traumatic brain injury. N Engl J Med 2012;367(26):2471-2481

16 Wijdicks EF, Hijdra A, Young GB, Bassetti CL, Wiebe S; Quality Standards Subcommittee of the American Academy of Neurology. Practice parameter: prediction of outcome in comatose survivors after cardiopulmonary resuscitation (an evidence-based review): report of the Quality Standards Subcommittee of the American Academy of Neurology. Neurology 2006;67(02):203-210

17 Friedman D, Claassen J, Hirsch LJ. Continuous electroencephalogram monitoring in the intensive care unit. Anesth Analg 2009; 109(02):506-523

18 Rossetti AO, Oddo M. The neuro-ICU patient and electroencephalography paroxysms: if and when to treat. Curr Opin Crit Care 2010;16(02):105-109

19 Kull LL, Emerson RG. Continuous EEG monitoring in the intensive care unit: technical and staffing considerations. J Clin Neurophysiol 2005;22(02):107-118

20 Hirsch LJ. Continuous EEG monitoring in the intensive care unit: an overview. J Clin Neurophysiol 2004;21(05):332-340

21 Roh DJ, Morris NA, Claassen J. Intracranial multimodality monitoring for delayed cerebral ischemia. J Clin Neurophysiol 2016; 33(03):241-249
22 Waziri A, Claassen J, Stuart RM, et al. Intracortical electroencephalography in acute brain injury. Ann Neurol 2009;66(03): 366-377

23 Claassen J, Perotte A, Albers D, et al. Nonconvulsive seizures after subarachnoid hemorrhage: multimodal detection and outcomes. Ann Neurol 2013;74(01):53-64

24 Claassen J, Vespa P; Participants in the International Multi-disciplinary Consensus Conference on Multimodality Monitoring. Electrophysiologic monitoring in acute brain injury. Neurocrit Care 2014;21(Suppl 2):S129-S147

25 Stuart RM, Schmidt M, Kurtz P, et al. Intracranial multimodal monitoring for acute brain injury: a single institution review of current practices. Neurocrit Care 2010;12(02):188-198

26 Hofmeijer J, Beernink TM, Bosch FH, Beishuizen A, TjepkemaCloostermans MC, van Putten MJ. Early EEG contributes to multimodal outcome prediction of postanoxic coma. Neurology 2015; 85(02):137-143

27 Claassen J, Mayer SA, Kowalski RG, Emerson RG, Hirsch LJ. Detection of electrographic seizures with continuous EEG monitoring in critically ill patients. Neurology 2004;62(10):1743-1748

28 Pandian JD, Cascino GD, So EL, Manno E, Fulgham JR. Digital videoelectroencephalographic monitoring in the neurological-neurosurgical intensive care unit: clinical features and outcome. Arch Neurol 2004;61(07):1090-1094

29 Sundt TM Jr, Sharbrough FW, Piepgras DG, Kearns TP, Messick JM Jr, O'Fallon WM. Correlation of cerebral blood flow and electroencephalographic changes during carotid endarterectomy: with results of surgery and hemodynamics of cerebral ischemia. Mayo Clin Proc 1981;56(09):533-543

30 Jordan KG. Emergency EEG and continuous EEG monitoring in acute ischemic stroke. J Clin Neurophysiol 2004;21(05):341-352

31 Simmons LE, Riker RR, Prato BS, Fraser GL. Assessing sedation during intensive care unit mechanical ventilation with the Bispectral Index and the Sedation-Agitation Scale. Crit Care Med 1999;27(08):1499-1504

32 Abou Khaled KJ, Hirsch LJ. Updates in the management of seizures and status epilepticus in critically ill patients. Neurol Clin 2008; 26(02):385-408, viii

33 Winer JW, Rosenwasser RH, Jimenez F. Electroencephalographic activity and serum and cerebrospinal fluid pentobarbital levels in determining the therapeutic end point during barbiturate coma. Neurosurgery 1991;29(05):739-741, discussion 741-742

34 Rossetti AO, Urbano LA, Delodder F, Kaplan PW, Oddo M. Prognostic value of continuous EEG monitoring during therapeutic hypothermia after cardiac arrest. Crit Care 2010;14(05):R173

35 Kassab MY, Majid A, Farooq MU, et al. Transcranial Doppler: an introduction for primary care physicians. J Am Board Fam Med 2007;20(01):65-71

36 Vora YY, Suarez-Almazor M, Steinke DE, Martin ML, Findlay JM. Role of transcranial Doppler monitoring in the diagnosis of cerebral vasospasm after subarachnoid hemorrhage. Neurosurgery 1999;44(06):1237-1247, discussion 1247-1248

37 Proust F, Callonec F, Clavier E, et al. Usefulness of transcranial color-coded sonography in the diagnosis of cerebral vasospasm. Stroke 1999;30(05):1091-1098

38 Bellner J, Romner B, Reinstrup P, Kristiansson KA, Ryding E, Brandt L. Transcranial Doppler sonography pulsatility index (PI) reflects intracranial pressure (ICP). Surg Neurol 2004;62(01):45-51, discussion 51

39 Feri M, Ralli L, Felici M, Vanni D, Capria V. Transcranial Doppler and brain death diagnosis. Crit Care Med 1994;22(07):1120-1126

40 Macmillan CS, Andrews PJ. Cerebrovenous oxygen saturation monitoring: practical considerations and clinical relevance. Intensive Care Med 2000;26(08):1028-1036

41 White H, Baker A. Continuous jugular venous oximetry in the neurointensive care unit-a brief review. Can J Anaesth 2002; 49(06):623-629 
42 Cruz J. On-line monitoring of global cerebral hypoxia in acute brain injury. Relationship to intracranial hypertension. J Neurosurg 1993;79(02):228-233

43 Cormio M, Valadka AB, Robertson CS. Elevated jugular venous oxygen saturation after severe head injury. J Neurosurg 1999; 90(01):9-15

44 Al-Rawi PG, Smielewski P, Kirkpatrick PJ. Evaluation of a near-infrared spectrometer (NIRO 300) for the detection of intracranial oxygenation changes in the adult head. Stroke 2001;32(11):2492-2500

45 Stiefel MF, Udoetuk JD, Spiotta AM, et al. Conventional neurocritical care and cerebral oxygenation after traumatic brain injury. J Neurosurg 2006;105(04):568-575

46 Gupta AK, Hutchinson PJ, Al-Rawi P, et al. Measuring brain tissue oxygenation compared with jugular venous oxygen saturation for monitoring cerebral oxygenation after traumatic brain injury. Anesth Analg 1999;88(03):549-553

47 Pfeifer R, Ferrari M, Börner A, Deufel T, Figulla HR. Serum concentration of NSE and S-100b during LVAD in non-resuscitated patients. Resuscitation 2008;79(01):46-53

48 Wolf H, Krall C, Pajenda G, et al. Preliminary findings on biomarker levels from extracerebral sources in patients undergoing trauma surgery: potential implications for TBI outcome studies. Brain Inj 2016;30(10):1220-1225

49 Brandner S, Thaler C, Buchfelder M, Kleindienst A. Brain-derived protein concentrations in the cerebrospinal fluid: contribution of trauma resulting from ventricular drain insertion. J Neurotrauma 2013;30(13):1205-1210
50 Ungerstedt $\mathrm{U}$, Rostami E. Microdialysis in neurointensive care. Curr Pharm Des 2004;10(18):2145-2152

51 Dreier JP, Major S, Manning A, et al; COSBID study group. Cortical spreading ischaemia is a novel process involved in ischaemic damage in patients with aneurysmal subarachnoid haemorrhage. Brain 2009;132(Pt 7):1866-1881

52 Tseng EE, Brock MV, Lange MS, et al. Glutamate excitotoxicity mediates neuronal apoptosis after hypothermic circulatory arrest. Ann Thorac Surg 2010;89(02):440-445

53 Nordmark J, Rubertsson S, Mörtberg E, Nilsson P, Enblad P. Intracerebral monitoring in comatose patients treated with hypothermia after a cardiac arrest. Acta Anaesthesiol Scand 2009; 53(03):289-298

54 Antunes AP, Schiefecker AJ, Beer R, et al. Higher brain extracellular potassium is associated with brain metabolic distress and poor outcome after aneurysmal subarachnoid hemorrhage. Crit Care 2014;18(03):R119

55 Nilsson OG, Brandt L, Ungerstedt U, Säveland H. Bedside detection of brain ischemia using intracerebral microdialysis: subarachnoid hemorrhage and delayed ischemic deterioration. Neurosurgery 1999;45(05):1176-1184, discussion 1184-1185

56 Misini B, Freinbichler W, Colivicchi MA, et al. Continuous monitoring of highly reactive oxygen radicals during in vivo microdialysis. J Neurosci Methods 2015;251:1-6

57 Helbok R, Schiefecker AJ, Beer R, et al. Early brain injury after aneurysmal subarachnoid hemorrhage: a multimodal neuromonitoring study. Crit Care 2015;19:75 